\title{
Expected Throughput of the Proportional Fair Scheduling over Rayleigh Fading Channels
}

\author{
Erwu Liu, Member, IEEE, and Kin K. Leung, Fellow, IEEE
}

\begin{abstract}
Existing analytical work on the proportional fair scheduling (PFS) algorithm has some limitations: 1) it requires independent identically distributed (i.i.d) relationship among users, 2) it uses a modified version of preference metric different from the original one, and 3 ) it is only valid for single-inputsingle-output (SISO) communications. This letter presents an analytical expression for the expected throughput of PFS over Rayleigh fading channels without the limitations above. The derived expression can be easily evaluated, and applies to both SISO and multiple-input-multiple-output (MIMO) cases. In addition, existing analytic result can be derived by using our expression in corresponding scenarios.
\end{abstract}

Index Terms-Proportional fairness, Rayleigh fading, multiinput-multi-output.

\section{INTRODUCTION}

$\mathbf{T}$ HE proportional fair scheduling (PFS) in wireless networks has gained lots of attention since its first present in [1] (see [2]-[4] and the references therein). However, one can only see limited analysis results on PFS [5]-[7]. Assuming the signal-to-noise ratio (SNR) follows an exponential distribution, [8] obtained the average throughput of PFS for two commonly-used rate models: linear rate model (i.e., rate $R_{i}=\beta \cdot S N R_{i}$ for any user $i$ ) and logarithm rate model (i.e., $\left.R_{i} \propto \log _{2}\left(1+\beta \cdot S N R_{i}\right)\right)$. Though [8] provides valuable results, two things should be pointed out: First, it uses a modified PFS metric other than the original one. Second, while the above-mentioned rate models ease the mathematical analysis, they are not satisfactory to characterize the feasible rate. In fact, the linear rate model is valid for very small SNR [5], while the logarithm rate model can only be used for singleinput-single-output (SISO) links. Moreover, to simplify the problem, most existing work [5]-[8] assume some kind of independent identically distributed (i.i.d) relationship among users. The i.i.d assumption and the use of a modified version of the preference metric are the two limitations in existing studies on PFS.

We have presented some results on PFS in previous work [4], [9], [10]. In [4], we analyzed PFS for SISO cases. In [9] we analyzed PFS for MIMO cases and extended to wireless mesh networks in [10]. All our previous work has a common limitation which requires the average moving factor $k$ (see Section II) to be infinitely large. Moreover, [4] assumes (expected throughput)/(expected feasible data rate) to be constant for all users in the analysis. Obviously, these potentially limit the practical use of our previous results.

Manuscript received January 11, 2010. The associate editor coordinating the review of this letter and approving it for publication was H. Wymeersch.

The authors are with the Department of Electrical and Electronic Engineering, Imperial College, London, United Kingdom (e-mail: \{erwu.liu, kkleung\}@ieee.org).

Digital Object Identifier 10.1109/LCOMM.2010.06.100048
For the analysis to be realistic, this letter analyzes the PFS performance for both SISO and MIMO links over Rayleigh fading channels without the limitations above.

\section{Mathematical Model And its Validity}

Consider a cellular network where there are $N$ users wishing to transmit to the base station (BS). The PFS algorithm is described as follows. Time is divided into small slots. In slot $m+1$, the feasible rate of User $j$ is $R_{j}[m+1]$. Its $k$-point moving average throughput up to slot $m$ is denoted by $\mu_{j}[m]$, and the preference metric by $M_{j}[m+1]=R_{j}[m+1] / \mu_{j}[m]$. User $i=\arg \max _{j} M_{j}[m+1]$ is scheduled to transmit in next slot. The moving average throughput of User $j$ is updated by

$$
\mu_{j}[m+1]=(1-1 / k) \mu_{j}[m]+I_{j}[m+1] \cdot R_{j}[m+1] / k .
$$

where $I_{j}[m+1]$ is the indicator function of the event that User $j$ is scheduled to transmit in slot $m+1$.

$$
I_{j}[m+1]=\left\{\begin{array}{ll}
1, & j \text { is scheduled in slot } m+1 \\
0, & \text { else }
\end{array} .\right.
$$

From (1), we have

$E\left[\mu_{j}[m+1]\right]=(1-1 / k) E\left[\mu_{j}[m]\right]+E\left[I_{j}[m+1] \cdot R_{j}[m+1]\right] / k$.

where $E[\cdot]$ denotes the statistical average.

Assuming first-order wide-sense stationary $\mu_{j}$ and applying Bayes's theorem, we can write (3) as

$$
\begin{aligned}
E\left[\mu_{j}[m]\right] & =E\left[R_{j}[m+1] \mid I_{j}[m+1]=1\right] \cdot \operatorname{Pr}\left(I_{j}[m+1]=1\right) \\
& =\int_{0}^{\infty} x f_{R_{j}}(x) \operatorname{Pr}\left(I_{j}[m+1]=1 \mid R_{j}[m+1]=x\right) d x(4)
\end{aligned}
$$

where $\operatorname{Pr}\left(I_{j}[m+1]=1\right)$ is the average probability that User $j$ will be scheduled in slot $m+1$, $\operatorname{Pr}\left(I_{j}[m+1]=1 \mid R_{j}[m+1]=x\right) \quad$ is $\quad$ the corresponding conditional probability and $f_{R_{j}}(\cdot)$ is the probability density function (pdf) of $R_{j}$.

From (1), we know that $\mu_{j}$ varies slowly with $R_{j}$ for large $k$, i.e., random variable $R_{j}[m+1] / \mu_{j}[m]$ can be approximately viewed as random variable $R_{j}[m+1] / E\left[\mu_{j}\right]$. In fact, experiments have suggested that this approximation is valid for $k \geq 50$ with an accuracy greater than $98 \%$. Hence we have,

$$
\begin{aligned}
& \operatorname{Pr}\left(I_{j}[m+1]=1 \mid R_{j}[m+1]=x\right) \\
& \quad \approx \operatorname{Pr}\left(\forall i \neq j, \frac{R_{i}[m+1]}{E\left[\mu_{i}\right]}<\frac{x}{E\left[\mu_{j}\right]}\right) .
\end{aligned}
$$

For statistically independent $R_{j}$,

$$
\begin{aligned}
& \operatorname{Pr}\left(I_{j}[m+1]=1 \mid R_{j}[m+1]=x\right) \\
& \approx \operatorname{Pr}\left(\forall i \neq j, R_{i}[m+1]<\frac{E\left[\mu_{i}\right]}{E\left[\mu_{j}\right]} x\right)=\prod_{i=1, i \neq j}^{N} F_{R_{i}}\left(\frac{E\left[\mu_{i}\right]}{E\left[\mu_{j}\right]} x\right) .(6)
\end{aligned}
$$


where $F_{R_{i}}(\cdot)$ is the cumulative distribution function (cdf) of $R_{i}$. Now we can rewrite (4) as,

$$
E\left[\mu_{j}\right]=\int_{0}^{\infty} x f_{R_{j}}(x) \prod_{i=1, i \neq j}^{N} F_{R_{i}}\left(E\left[\mu_{i}\right] \cdot x / E\left[\mu_{j}\right]\right) d x .
$$

Simulations (see Fig. 2) have found that, for PFS over Rayleigh fading channels, $\sigma_{R_{i}} / \sigma_{R_{j}} \leq E\left[\mu_{i}\right] / E\left[\mu_{j}\right] \leq$ $E\left[R_{i}\right] / E\left[R_{j}\right]$ given $0<E\left[R_{j}\right] \leq E\left[R_{i}\right]$, where $\sigma_{R_{i}}$ is the standard deviation of $R_{i}$. Denote $E\left[R_{j}\right] / \sigma_{R_{j}}$ by $M_{j}$, we have

$$
\begin{aligned}
& E\left[\mu_{j}\right] \geq \sigma_{R_{j}} \int_{-M_{j}}^{\infty}\left(y \sigma_{R_{j}}+E\left[R_{j}\right]\right) \cdot f_{R_{j}}\left(y \sigma_{R_{j}}+E\left[R_{j}\right]\right) \\
& \times \prod_{\forall i \neq j, E\left[R_{i}\right]<E\left[R_{j}\right]}^{N} F_{R_{i}}\left(y \frac{E\left[R_{i}\right]}{E\left[R_{j}\right]} \sigma_{R_{j}}+E\left[R_{i}\right]\right) \\
& \times \prod_{\forall i \neq j, E\left[R_{i}\right] \geq E\left[R_{j}\right]}^{N} F_{R_{i}}\left(y \sigma_{R_{i}}+\frac{\sigma_{R_{i}}}{\sigma_{R_{j}}} E\left[R_{j}\right]\right) d y . \quad(8) \\
& E\left[\mu_{j}\right] \leq \sigma_{R_{j}} \int_{-M_{j}}^{\infty}\left(y \sigma_{R_{j}}+E\left[R_{j}\right]\right) \cdot f_{R_{j}}\left(y \sigma_{R_{j}}+E\left[R_{j}\right]\right) \\
& \times \quad \prod_{\forall i \neq j, E\left[R_{i}\right] \geq E\left[R_{j}\right]}^{N} F_{R_{i}}\left(y \frac{E\left[R_{i}\right]}{E\left[R_{j}\right]} \sigma_{R_{j}}+E\left[R_{i}\right]\right) \\
& \times \quad \prod_{\forall i \neq j, E\left[R_{i}\right]<E\left[R_{j}\right]}^{N} F_{R_{i}}\left(y \sigma_{R_{i}}+\frac{\sigma_{R_{i}}}{\sigma_{R_{j}}} E\left[R_{j}\right]\right) d y . \quad(9)
\end{aligned}
$$

Since $F_{R}(\cdot)$ is non-decreasing and $\sigma_{R_{i}} / \sigma_{R_{j}} \leq E\left[R_{i}\right] / E\left[R_{j}\right]$ given $0<E\left[R_{j}\right] \leq E\left[R_{i}\right]$, we can prove that (10) lies between the lower and upper bounds given by (8) and (9),

$$
\begin{aligned}
\sigma_{R_{j}} \int_{-M_{j}}^{\infty}\left(y \sigma_{R_{j}}\right. & \left.+E\left[R_{j}\right]\right) \cdot f_{R_{j}}\left(y \sigma_{R_{j}}+E\left[R_{j}\right]\right) \\
& \times \prod_{i=1, i \neq j}^{N} F_{R_{i}}\left(y \sigma_{R_{i}}+E\left[R_{i}\right]\right) d y .
\end{aligned}
$$

This is our approximation of the expected PFS throughput.

Smith and McKay et al. [11], [12] have pointed out that the feasible rate of a SISO or MIMO link over Rayleigh fading channels can be modeled by a Gaussian distribution with surprisingly high accuracy. We will show in the following that such property turns (10) into a simple closed-form expression.

It is known that for a Gaussian distributed random variable $Z$ with expect value $E[Z]$ and standard deviation $\sigma_{Z}$, we have,

1) $f_{Z}(z)=f_{(0,1)}\left((z-E[Z]) / \sigma_{Z}\right) / \sigma_{Z}$,

2) $F_{Z}(z)=F_{(0,1)}\left((z-E[Z]) / \sigma_{Z}\right)$.

where $f_{(0,1)}(\cdot)$ and $F_{(0,1)}(\cdot)$ are the pdf and cdf of zero mean, unit variance standard normal distribution, respectively.

As stated earlier, the feasible rate in a Rayleigh environment can be approximately modeled by a Gaussian distribution. With the above two properties we can rewrite (10) as

$$
\begin{aligned}
& E\left[\mu_{j}\right] \approx \int_{-M_{j}}^{\infty}\left(y \sigma_{R_{j}}+E\left[R_{j}\right]\right) \cdot f_{(0,1)}(y) \cdot \prod_{i=1, i \neq j}^{N} F_{(0,1)}(y) d y \\
&=E\left[R_{j}\right] / N \cdot\left(1-\left[F_{(0,1)}\left(-E\left[R_{j}\right] / \sigma_{R_{j}}\right)\right]^{N}\right) \\
& \quad+\sigma_{R_{j}} \int_{-M_{j}}^{\infty} y f_{(0,1)}(y) \cdot\left[F_{(0,1)}(y)\right]^{N-1} d y \cdot(11)
\end{aligned}
$$

Unlike existing work, our analysis replaces the requirement for an identical distribution with the assumption of a Gaussian distribution, but note that independence is still necessary.

Formula (11) has a very clear physical meaning: the first item in the right-hand side (RHS) represents the average throughput from round-robin (RR) scheduling, $E\left[R_{j}\right] / N$ (note $\left.\left[F_{(0,1)}\left(-E\left[R_{j}\right] / \sigma_{R_{j}}\right)\right]^{N} \approx 0\right)$, while the second item represents the average throughput from fading variability.

We now conduct simulations for various fading scenarios to verify our theoretical results. Simulation setups are

1) Simulation parameters: 4000 scheduling slots, moving average factor $k=500$.

2) A network of 10 users with average $S N R=1,2, \cdots, 10$ $\mathrm{dB}$, respectively, where users have independent Rayleigh fading. For the simplicity of presentation, each user is numbered by its average $S N R$. We consider two cases.

Case I: SISO is used between a user and the BS.

Case II: $5 \times 5$ MIMO is used between a user and the BS.

According to [13], for a $t \times r$ MIMO link over Rayleigh channel, where $t$ and $r$ are the numbers of transmit and receive antennas, there are $m=\min (t, r)$ nonnegative singular values of the channel matrix, each with the same pdf

$$
p(\lambda)=\frac{1}{m} \cdot \sum_{i=0}^{m-1} i ! \lambda^{n-m} e^{-\lambda}\left[L_{i}^{n-m}(\lambda)\right]^{2} /(i+n-m) !
$$

where $n=\max (t, r), L_{k}^{n-m}(\cdot)$ is generalized Laguerre polynomials of order $k$. Each nonnegative singular value corresponds to a parallel/independent channel. In the simulation, we assume each sender allocates its power equally to all the transmit antennas and we generate the feasible rate of a MIMO link by $R=m \cdot \log _{2}(1+\overline{S N R} \cdot \lambda / t)$, where $\overline{S N R}$ is the average SNR, $\lambda$ is a random variable with the pdf given by (12).

We use (11) to numerically calculate the average throughput of PFS, where $E[R]$ and $\sigma_{R}$ are determined by [11], [13]

$$
\begin{gathered}
E[R]=m \int_{0}^{\infty} \omega(\lambda) p(\lambda) d \lambda . \\
\sigma_{R}^{2}=m \int_{0}^{\infty} \omega^{2}(\lambda) p(\lambda) d \lambda-\sum_{i=0}^{m-1} \sum_{j=0}^{m-1} \frac{i ! j !}{(i+n-m) !(j+n-m) !} \\
\times\left(\int_{0}^{\infty} \lambda^{n-m} e^{-\lambda} L_{i}^{n-m}(\lambda) L_{j}^{n-m}(\lambda) \omega(\lambda) d \lambda\right)^{2}
\end{gathered}
$$

where $\omega(\lambda)=\log _{2}(1+\overline{S N R} \cdot \lambda / t)$.

Figs. 1 and 2 depict the simulation and theoretical results for Case I. Fig. 1 validates that both our analytical expression and the existing result [8] provide accurate estimate of the PFS throughput for SISO cases.

Fig. 2 shows that, for PFS over Rayleigh fading channels, it satisfies $\sigma_{R_{i}} / \sigma_{R_{j}} \leq E\left[\mu_{i}\right] / E\left[\mu_{j}\right] \leq E\left[R_{i}\right] / E\left[R_{j}\right]$, given $0<$ $E\left[R_{j}\right] \leq E\left[R_{i}\right]$ (from (13) and (14), it is easy to prove that both $E[R]$ and $\sigma_{R}$ are monotonically increasing function of average $S N R$ ). In addition, Fig. 2 indicates that the throughput gain of PFS over RR scheduling (i.e., gain $G=N \cdot E[\mu] / E[R] \propto$ $\sigma_{R} / E[R]$ according to (11), where $N$ is the number of users in the network) for a bad-channel user (low $E[R]$ ) is higher than a good-channel user (high $E[R]$ ). Mathematically, by using 


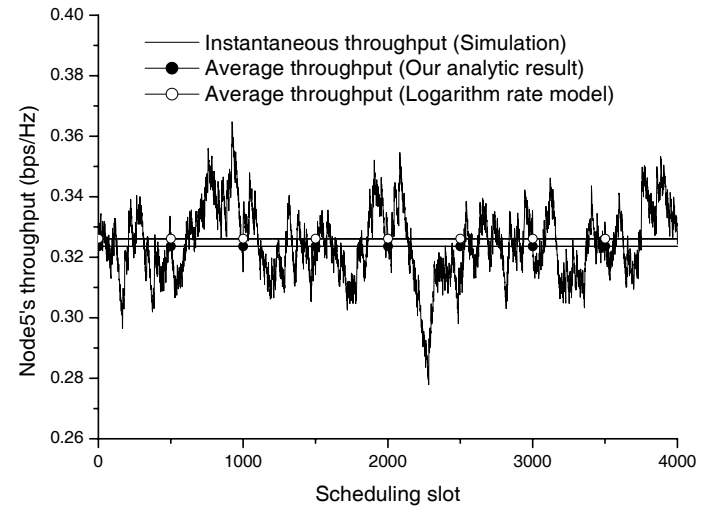

Fig. 1. PFS throughput (Case I: SISO).

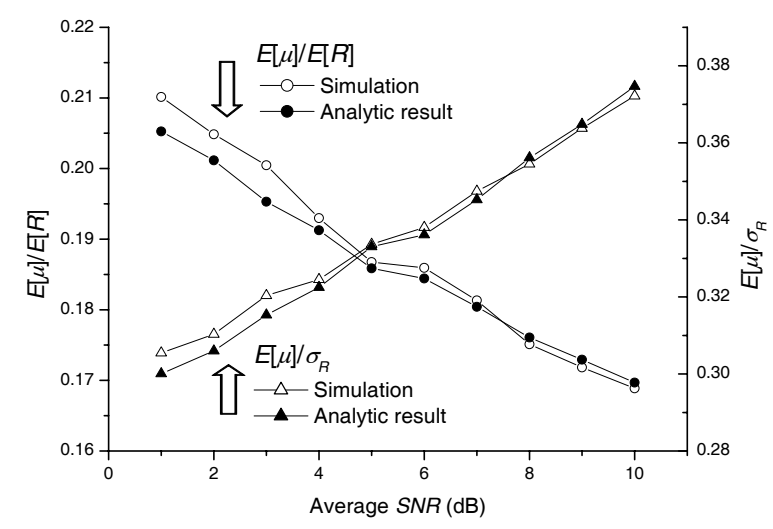

Fig. 2. $E[\mu] / E[R], E[\mu] / \sigma_{R}$ w.r.t $S N R$.

(13) and (14) one can prove $\sigma_{R} / E[R]$ decreases with $E[R]$ in Rayleigh fading environments to explain this phenomenon. To put it simple: in RR scheduling, bad-channel users will suffer from very low throughput, whereas PFS, through multi-user diversity, compensates bad-channel users by 'stealing' some throughput benefits from good-channel users.

Fig. 3 depicts the simulation and theoretical results for Case II. Fig. 3 shows that our analytic formula also applies to MIMO cases while the existing result under the logarithm rate model [8] is not satisfactory in such scenarios. The difference is due to the fact that while the logarithm rate model can be used to describe the feasible rate of a SISO link, it is not accurate in modeling the feasible rate of a MIMO link [13].

\section{CONCLUSIONS}

This work derives mathematical models for the PFS throughput under Rayleigh fading environments. The analytical expressions are validated by simulations. Moreover, comparisons between our result and the existing one are given, which suggest that our results apply to both SISO and MIMO links while the existing result is only valid for SISO cases.

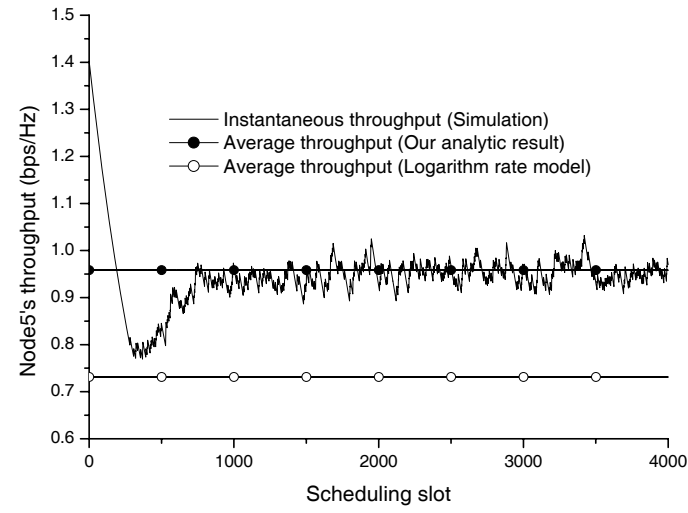

Fig. 3. PFS throughput (Case II: MIMO).

\section{REFERENCES}

[1] F. Kelly, "Charging and rate control for elastic traffic," Europ. Trans. Telecommun., vol. 8, pp. 33-37, Feb. 1997.

[2] P. Viswanath, D. N. C. Tse, and R. Laroia, "Opportunistic beamforming using dumb antennas," IEEE Trans. Inf. Theory, vol. 48, pp. 1277-1294, 2002.

[3] T.-D. Nguyen and Y. Han, "A proportional fairness algorithm with QoS provision in downlink OFDMA systems," IEEE Commun. Lett., vol. 10, pp. 760-762, Nov. 2006.

[4] E. Liu and K. K. Leung, "Proportional fair scheduling: analytical insight under Rayleigh fading environment," in Proc. IEEE Wireless Communications and Networking Conference (WCNC 2008), pp. 18831888, Mar. 2008.

[5] J. M. Holtzman, "Asymptotic analysis of proportional fair algorithm," in Proc. 12th IEEE International Symposium on Personal, Indoor and Mobile Radio Communications (PIMRC 2001), vol. 2, pp. 33-37, Sep. 2001.

[6] S. Borst, "User-level performance of channel-aware scheduling algorithms in wireless data networks," in Proc. INFOCOM 2003, vol. 1, pp. 321-331, Mar. 2003.

[7] D. Avidor, S. Mukherjee, J. Ling, and C. Papadias, "On some properties of the proportional fair scheduling policy," in Proc. 15th IEEE International Symposium on Personal, Indoor and Mobile Radio Communications (PIMRC 2004), vol. 2, pp. 853-858, Sep. 2004.

[8] J.-G. Choi and S. Bahk, "Cell-throughput analysis of the proportional fair scheduler in the single-cell environment," IEEE Trans. Veh. Technol., vol. 56, pp. 766-778, Mar. 2007.

[9] E. Liu and K. K. Leung, "Fair resource allocation under Rayleigh and/or Rician fading environments," in Proc. IEEE 19th International Symposium on Personal, Indoor and Mobile Radio Communications (PIMRC 2008), pp. 1-5, Sep. 2008.

[10] E. Liu and K. K. Leung, "On proportional fair scheduling in multiantenna wireless mesh networks-theoretical analysis," in Proc. IEEE Global Telecommunications Conference (GLOBECOM 2008), pp. 168 172, Nov. 2008.

[11] P. J. Smith and M. Shafi, "On a Gaussian approximation to the capacity of wireless MIMO systems," in Proc. IEEE International Conference on Communications (ICC 2002), vol. 1, pp. 406-410, Apr. 2002.

[12] M. R. McKay, P. J. Smith, H. A. Suraweera, and I. B. Collings, "On the mutual information distribution of OFDM-based spatial multiplexing: exact variance and outage approximation," IEEE Trans. Inf. Theory, vol. 54, pp. 3260-3278, July 2008.

[13] I. E. Telatar, "Capacity of multi-antenna Gaussian channels," Europ. Trans. Telecommun., vol. 10, pp. 585-595, Nov. 1999. 